ORIGINAL ARTICLE

\title{
Synergistic effect of hepatitis virus infection and occupational exposures to vinyl chloride monomer and ethylene dichloride on serum aminotransferase activity
}

\author{
H-I Hsieh, J-D Wang, P-C Chen, T-J Cheng
}

Occup Environ Med 2003;60:774-778

See end of article for authors' affiliations

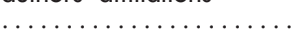

Correspondence to: Dr T-J Cheng, Institute of Occupational Medicine and Industrial Hygiene, College of Public Health National Taiwan University, Taipei, Taiwan 10018:

tcheng@ha.mc.ntu.edu.tw

Accepted

21 September 2002

\begin{abstract}
Aims: To study the synergistic effect of occupational chemical exposure and hepatitis virus infection on serum aminotransferase activity.

Methods: A total of 568 male workers who were employed in five polyvinyl chloride (PVC) or four vinyl chloride monomer (VCM) manufacturing factories were studied. Information relating to current job title, alcohol consumption, and cigarette smoking was obtained. Exposure level of chemical mixtures was classified by hygienic effect (a summation of personal time weighted average/reference permissible exposure level of each chemical) into high, moderate, and low exposure groups. Serum aspartate aminotransferase (AST) and alanine aminotransferase (ALT), hepatitis B surface antigen (HBsAg), hepatitis $B$ e antigen $(\mathrm{HBeAg})$, and anti-hepatitis $C$ antibody were assayed.

Results: Hepatitis virus infection and increased body mass index were associated with abnormal serum aminotransferase activity. In workers with hepatitis virus infection, those with high exposure had a higher prevalence of abnormal AST and ALT compared to low exposure; among those without hepatitis virus infection, the differences of prevalence of abnormal AST and ALT were not significant between different chemical exposure groups. There was a significant trend of increasing risks of increased AST and ALT in moderate and high exposure groups with hepatitis virus infection. Such a synergistic effect was more prominent among $\mathrm{HBeAg}$-positive workers.

Conclusions: Mixed exposures to 1,2-ethylene dichloride and VCM have a positive synergistic effect with hepatitis virus infection on liver damage. Assessment of fitness for work should be considered in workers with hepatitis $B$ and $C$ infection, when they have potential exposure to hepatotoxins in the workplace.
\end{abstract}

$M$ easurements of serum aspartate aminotransferase (AST) and alanine aminotransferase (ALT) have been used extensively in the assessment of liver damage. Abnormal serum aminotransferases have been associated with occupational and non-occupational factors. ${ }^{1}$ Among non-occupational hepatotoxins, hepatitis $\mathrm{B}$ and $\mathrm{C}$ virus infection have synergistic effects with alcohol consumption on serum aminotransferases. ${ }^{23}$ However, it is not clear whether occupational chemical exposure and hepatitis B and $\mathrm{C}$ have synergistic effects on these hepatic enzymes or not. This question needs to be resolved for the assessment of fitness of work, particularly in populations with a high prevalence of hepatitis B infection, including Taiwan. ${ }^{45}$

We have studied liver disorders in vinyl chloride monomer (VCM) and polyvinyl chloride (PVC) manufacturing workers over the past decade. VCM (CAS no. 75-01-4) or 1,2-ethylene dichloride (EDC; CAS no. 107-06-2) exposure has been associated with serum aminotransferase abnormalities in our previous studies. ${ }^{67}$ In the above studies, it seems that exposed workers with hepatitis B infection tend to have a higher risk of abnormal serum aminotransferase compared to those without hepatitis B infection. However, it was difficult to draw a conclusion because of the small number of subjects with abnormal serum aminotransferase. To increase the power of detection for the interaction between hepatitis virus infection and occupational chemical exposure, we included workers from both PVC and VCM manufacturing plants, who received medical examinations between 1995 and 1997. Since workers with hepatitis B e antigen (HBeAg) also had a higher prevalence of serum aminotransferase abnormality, ${ }^{8}$ the interaction between $\mathrm{HBeAg}$ and occupational chemical exposure on serum aminotransferase was further assessed.

\section{METHODS}

Study population

A total of 617 workers were eligible. Because 49 workers had incomplete data, 568 (92\%) workers from five PVC $(\mathrm{n}=292)$ and four VCM $(\mathrm{n}=276)$ manufacturing factories were included for the analysis. Among them, 11 workers were office workers, who stayed indoor during most of their working hours, and three workers were guards who stayed away from the manufacturing site. Most of these workers have been presented in previous studies to investigate the relation between external chemical exposures and liver function or genotoxicity, or the effects of metabolic genotypes on liver function or genotoxicity. ${ }^{679}$

After informed consent was obtained, all study subjects were surveyed by an interviewer administered questionnaire to obtain information on smoking, alcohol consumption, medicines, and medical and occupational histories. Alcohol consumption was calculated from drinking frequency and alcohol content of each beverage consumed. Those who drank at least once and had alcohol consumption with a

\footnotetext{
Abbreviations: anti-HCV, anti-hepatitis $\mathrm{C}$ antibody; $\mathrm{ALT}$, alanine aminotransferase; AST, aspartate aminotransferase; BMI, body mass index; EDC, 1,2-ethylene dichloride; GSH, glutathione; $\mathrm{HBeAg}$, hepatitis $B$ e antigen; $\mathrm{HBsAg}$, hepatitis $B$ surface antigen; $\mathrm{PEL}$, permissible exposure level; PVC, polyvinyl chloride; TWA, time weighted average; VCM, vinyl chloride monomer
} 
Main messages

- Hepatitis virus infection and increased body mass index are important non-occupational factors that increase serum aminotransferase in asymptomatic chemical workers.

- Hepatitis virus infection has a synergistic effect with exposure to vinyl chloride monomer and 1,2-ethylene dichloride on serum aminotransferase activity.

- The synergistic effect of hepatitis virus infection and chemical exposures on serum aminotransferase is more prominent in workers with positive $\mathrm{HBeAg}$ compared to those with positive $\mathrm{HBsAg}$ but without $\mathrm{HBeAg}$.

minimum of $80 \mathrm{~g}$ intake per week in the past one month were defined as having drinking habit. Smoking behaviour was defined as having smoked at least one cigarette per day within the preceding six months of data collection.

\section{Exposure assessment}

EDC is used in the production of VCM, and VCM is subsequently used for the polymerisation to manufacture PVC. In VCM manufacturing plants, workers were exposed to both EDC and VCM, while workers in PVC plants were exposed to VCM only. Detailed occupational history included job title, daily activity, and use of respirators in the current and previous jobs. Personal samplings were conducted to calculated EDC and VCM time weighted average (TWA) for each category of work. ${ }^{10}$ If personal sampling data were not available, data of area sampling was used. Office workers and guards were presumably exposed to extreme low concentrations of chemicals, thus $0 \mathrm{ppm}$ of VCM and EDC were assumed as their TWA. To consider the combined effect of EDC and VCM, the hygienic effect was calculated by using the model of $\left(C_{1} / T_{1}\right)+\left(C_{2} / T_{2}\right)$, where $C$ was the measured TWA and $T$ was the permissible exposure levels or equivalents for each chemical. One ppm was used for both EDC and VCM in this study, which has been adopted by many institutions. Our previous study also found that EDC and VCM cause abnormal liver aminotransferase around 1 ppm. ${ }^{7}$ Workers with hygienic effects below 1 were classified into the low exposure group. Workers with hygienic effects between 1 and 5 were classified into the moderate exposure group, and workers with exposures greater than 5 were classified into the high exposure group.

\section{Biochemical tests and hepatitis virus markers}

Markers of liver damage, including AST and ALT, were analysed with a Hitachi 7050 autoanalyser (Hitachi Co., Tokyo, Japan) at National Taiwan University Hospital (NTUH). Hepatitis B virus surface antigen (HBsAg) and anti-hepatitis $\mathrm{C}$ virus antibody (anti-HCV) were determined by enzyme linked immunoassay (EIA, Abbott Laboratories, Chicago, IL, USA), respectively. HBeAg was also assayed by ELISA in workers with positive HBsAg. Abnormal results for serum aminotransferases were defined as having values greater than the reference provided by NTUH. In 1995, it was 31 for both ALT and AST. In 1996 and 1997, it was 37 for AST, and 41 for ALT. Subjects with positive hepatitis B infection were defined as having positive HBsAg, and subjects with positive hepatitis $\mathrm{C}$ infection were defined as having positive anti-HCV. Since the number of subjects exhibiting a positive titre for anti-HCV was small, HBsAg and anti-HCV were grouped together as hepatitis virus infection. Body mass

\section{Policy implications}

- Assessment of fitness for work should be considered in workers with hepatitis B and C infection, when they have potential exposure to hepatotoxins in the workplace.

- In countries where hepatitis $B$ and $C$ virus infection is prevalent, a more stringent occupational standard is needed to protect workers exposed to hepatotoxins.

index (BMI) was calculated as weight in kilograms divided by the square of height in metres.

\section{Statistical analysis}

The PC/SAS statistical package (SAS Institute Inc., Cary, NC, USA) was used for the statistical analysis. The $\chi^{2}$ test was used to compare the differences of age, employment duration, hepatitis virus infections, body mass index, and alcohol consumption between different exposure groups. Crude comparisons of abnormal AST and ALT by variables of interests were conducted in the univariate analysis. Subsequently, a multiple logistic regression model was used to determine the odds ratio (OR) of abnormal AST and ALT levels for different exposure groups (high, moderate, and low chemical exposure), hepatitis virus infection (yes and no), body mass index $\left(\geqslant 25.0\right.$ and $\left.<25.0 \mathrm{~kg} / \mathrm{m}^{2}\right)$, and habitual drinking (yes and no). OR of abnormal AST and ALT levels on different exposure groups stratified by hepatitis B and C virus infection was also calculated after controlling for potential confounders including age, BMI, and alcohol drinking. OR of abnormal AST and ALT on the chemical exposure (low, moderate, and high) was further calculated by HBeAg and HBsAg status $(-/-,-/+$, and $+/+$, respectively). All $p$ values were quoted two sided, and those values $<0.05$ were regarded as statistically significant.

\section{RESULTS}

\section{Descriptive statistics}

Table l summarises the basic characteristics of study subjects stratified by different exposure groups. The median TWA of VCM was 0.67 (range 0.0-73.8) ppm and of EDC was 0.35 (range 0.0-30.5) ppm. Most workers (83.6\%) were less than 50 years of age, $29.8 \%$ of workers had BMI greater than 25 , $11.1 \%$ of workers consumed more than $80 \mathrm{~g}$ of alcohol per week, $17.3 \%$ of workers had HBsAg, and $3.5 \%$ of workers had HBeAg. The high exposure group had more habitual drinkers than the moderate and low exposure groups. The low exposure group was older than the moderate and high exposure groups. All other characteristics of cigarette smoking, BMI, HBeAg, HBsAg, and Anti-HCV were not statistically significant between these three exposure groups.

Overall, 112 workers (19.7\%) showed increases of AST or ALT. There were $22.4 \%, 20.4 \%$, and $18.6 \%$ of workers with abnormal AST or ALT among the high, moderate, and low exposure groups. Workers with high exposure had more cases of abnormal AST or ALT when compared with other workers, but this did not reach statistical significance.

\section{Multiple logistic regression analysis}

Multiple logistic regression analysis (see table 2) revealed that AST was associated with BMI and hepatitis virus infection. There were similar findings with ALT, but the association with hepatitis C infection and ALT did not reach statistical significance. Increased chemical exposure was also associated with abnormal ALT or AST, but this association did not reach statistical significance. 


\begin{tabular}{|c|c|c|c|c|}
\hline \multirow[b]{2}{*}{ Characteristic } & \multicolumn{4}{|c|}{ Chemical exposure } \\
\hline & $\begin{array}{l}\text { High }(\geqslant 5) \\
(n=76)(\%)\end{array}$ & $\begin{array}{l}\text { Moderate }(\geqslant 1 \text { and } \\
<5)(n=191)(\%)\end{array}$ & $\begin{array}{l}\text { Low }(<1) \\
(n=301)(\%)\end{array}$ & $\begin{array}{l}\text { Total } \\
(n=568)(\%)\end{array}$ \\
\hline \multicolumn{5}{|l|}{ Manufacturing factory } \\
\hline PVC & 67.1 & 64.4 & 39.2 & $51.4^{* *}$ \\
\hline VCM & 32.9 & 25.6 & 60.8 & $48.6^{* \star}$ \\
\hline Age $\geqslant 40$ years & 46.1 & 46.1 & 59.1 & $53.0^{*}$ \\
\hline Duration of employment $\geqslant 15$ years & 43.4 & 45.0 & 56.5 & $50.9^{*}$ \\
\hline Body mass index $\geqslant 25.0 \mathrm{~kg} / \mathrm{m}^{2}$ & 29.0 & 33.0 & 27.9 & 29.8 \\
\hline Current cigarette smoking (yes) & 48.7 & 43.5 & 36.9 & 40.7 \\
\hline Alcohol drinking (yes) & 23.7 & 8.4 & 9.6 & $11.1^{* *}$ \\
\hline Positive hepatitis B surface antigen ( $\mathrm{HBs} A \mathrm{Ag})$ & 19.7 & 15.7 & 17.6 & 17.3 \\
\hline Positive hepatitis $\mathrm{B}$ e antigen ( $\mathrm{HBeAg}$ ) & 7.9 & 1.6 & 3.7 & 3.5 \\
\hline Positive anti-hepatitis $\mathrm{C}$ antibody (anti-HCV) & 1.3 & 4.7 & 3.7 & 3.7 \\
\hline
\end{tabular}

\section{Interaction analysis of factors on serum aminotransferase}

The interactions between chemical exposure and each potential factor (hepatitis virus infection, BMI, and alcohol drinking) on serum aminotransferase were calculated. Significant interactions were observed for chemical exposure and hepatitis virus infection (table 3). When workers did not have hepatitis virus infection, all serum aminotransferases showed no difference among different exposure groups. Dose dependent effects of chemicals on AST and ALT were found when workers had hepatitis virus infection. Workers who had high chemical exposure and hepatitis virus infection had the highest risk of abnormal AST (OR 10.6; 95\% CI 3.6 to 31.5) and ALT (OR 6.4; 95\% CI 2.1 to 19.1) when compared with workers who had low chemical exposure and lacked hepatitis virus infection. If we confined the analysis to those with hepatitis virus infection, the high exposure group had the highest risk of abnormal AST (OR 6.2; 95\% CI 1.8 to 21.4) and ALT (OR 6.5; 95\% CI 1.8 to 23.6) when compared with the low exposure group.

Further analysis was also performed when workers with positive HBsAg were divided into two groups according to the presence of HBeAg status (fig 1). After adjustment for age, drinking, and BMI, significant higher risks of abnormal AST (OR 7.1; 95\% CI 0.6 to 81.2) and ALT (OR 21.0; 95\% CI 1.4 to 320.7) were observed in the high exposure group compared with the low exposure group among workers with both HBsAg and HBeAg. Among workers who had positive HBsAg but did not have positive HBeAg, a higher risk of abnormal AST (OR 5.1; 95\% CI 1.1 to 24.3 ) or ALT (OR 3.7; 95\% CI 0.7 to 20.0) was observed in the high exposure group when compared with the low exposure group. Moreover, among workers without HBsAg, chemical exposure did not increase the risk of abnormal liver function. Workers who had positive HBeAg and high chemical exposure had the highest risk of abnormal AST (OR 29.5; 95\% CI 5.2 to 166.0) and ALT (OR 25.6 ; $95 \%$ CI 3.9 to 168.0 ) when compared with low exposed workers without HBsAg. There was a statistically significant trend (Mantel extension test for trend, $p<0.01$ ) for increased frequency of abnormal AST and ALT together with severity of hepatitis virus infection among the high exposure group.

Among workers who consumed alcohol or not, prevalence of abnormal AST and ALT was not observed between different chemical exposure groups. It is interesting to observe that, after adjusted for hepatitis virus infection, age, and BMI, drinkers with hepatitis virus infection had higher rates of abnormal AST (OR 9.1; 95\% CI 2.4 to 34.1) and ALT (OR 2.9; $95 \%$ CI 0.9 to 10.1) compared to those without hepatitis virus infection and habitual drinking. The positive interaction effect of hepatitis virus infection and alcohol consumption on AST reached statistical significance.

\section{DISCUSSION}

The results show that occupational chemical exposure had a positive interaction with hepatitis B and/or C infection on serum AST and ALT. This synergistic effect was most obvious in workers with positive HBeAg. However, a statistical association may not indicate a causal association. As the most commonly reported determinants for increase of AST and/or ALT, including BMI, alcohol, and age, were all controlled, we suspected that such an association might be causal.

Table 2 Odds ratios (OR) with 95\% confidence intervals (CI) of multiple logistic regression modelling adjusted for major determinants, including body mass index (BMI), chemical exposure, hepatitis B surface antigen $(\mathrm{HBsAg})$, anti-hepatitis $C$ antibody (Anti-HCV), drinking, and smoking

\begin{tabular}{|c|c|c|c|}
\hline \multirow[b]{2}{*}{ Determinants } & \multirow[b]{2}{*}{ Definition } & \multirow{2}{*}{$\begin{array}{l}\text { AST } \\
\text { OR }(95 \% \mathrm{CI})\end{array}$} & \multirow{2}{*}{$\begin{array}{l}\text { ALT } \\
\text { OR }(95 \% \mathrm{CI})\end{array}$} \\
\hline & & & \\
\hline Age (years) & $\geqslant 40.0 v<40.0$ & $0.8(0.5$ to 1.5$)$ & $0.6(0.4$ to 1.0$)$ \\
\hline $\mathrm{BMI}\left(\mathrm{kg} / \mathrm{m}^{2}\right)$ & $\geqslant 25.0 v<25.0$ & $2.2(1.2 \text { to } 3.9)^{*}$ & $3.5(2.2 \text { to } 5.5)^{*}$ \\
\hline \multirow[t]{2}{*}{ Chemical exposure } & High $v$ low & $1.3(0.6$ to 2.9$)$ & $1.4(0.7$ to 2.6$)$ \\
\hline & Moderate $v$ low & $0.8(0.4$ to 1.5$)$ & $1.0(0.6$ to 1.6$)$ \\
\hline $\mathrm{HBsAg}$ & Positive $v$ negative & $3.5(1.9 \text { to } 6.4)^{*}$ & $2.5(1.5 \text { to } 4.2)^{*}$ \\
\hline Anti-HCV & Positive $v$ negative & $5.9(2.2 \text { to } 15.9)^{*}$ & $2.3(0.8$ to 6.3$)$ \\
\hline Drinking & Yes $v$ no & $1.3(0.6$ to 3.0$)$ & $0.9(0.5$ to 1.9$)$ \\
\hline Smoking & Yes $v$ no & $1.1(0.6$ to 2.1$)$ & $1.0(0.6$ to 1.6$)$ \\
\hline
\end{tabular}


Table 3 Frequencies and adjusted odds ratios (OR) with 95\% confidence intervals (CI) of abnormal serum aminotransferase activity stratified by exposure classification and hepatitis virus infection; all odds ratios were adjusted for age, body mass index, and alcohol drinking

\begin{tabular}{|c|c|c|c|c|c|c|}
\hline \multirow[b]{2}{*}{ Category } & \multicolumn{3}{|c|}{ AST $(n=58)$} & \multicolumn{3}{|c|}{ ALT $(n=103)$} \\
\hline & $\%$ & OR $(95 \% \mathrm{Cl}) \dagger$ & OR $(95 \% \mathrm{Cl}) \ddagger$ & $\%$ & OR $(95 \% \mathrm{Cl}) \dagger$ & OR $(95 \% \mathrm{Cl}) \ddagger$ \\
\hline \multicolumn{7}{|c|}{$\mathrm{HBsAg}(+)$ or anti-HCV (+) } \\
\hline High exposure & 56.3 & $10.6(3.6 \text { to } 31.5)^{*}$ & $6.2(1.8 \text { to } 21.4)^{*}$ & 62.5 & $6.4(2.1 \text { to } 19.1)^{*}$ & $6.5(1.8 \text { to } 23.6)^{*}$ \\
\hline Moderate exposure & 23.5 & $3.0(1.1$ to 8.0$)$ & $1.6(0.4$ to 5.7$)$ & 35.3 & $2.1(0.9$ to 5.0$)$ & $2.3(0.7$ to 7.5$)$ \\
\hline Low exposure & 15.9 & $2.3(0.9$ to 5.4$)$ & 1.0 (referent) & 19.0 & $1.4(0.7$ to 3.0$)$ & 1.0 (referent) \\
\hline \multicolumn{7}{|c|}{ HBsAg $(-)$ and anti-HCV $(-)$} \\
\hline High exposure & 1.7 & $0.5(0.1$ to 1.5$)$ & & 11.7 & $0.5(0.2$ to 1.3$)$ & \\
\hline Moderate exposure & 5.7 & $0.6(0.3$ to 1.6$)$ & & 14.6 & $0.7(0.4$ to 1.3$)$ & \\
\hline Low exposure & 8.8 & 1.0 (referent) & & 16.4 & 1.0 (referent) & \\
\hline
\end{tabular}

${ }^{*} \mathrm{p}<0.01$ by Mantel-Haenszel $\chi^{2}$ test for trend analysis.

†The referent group $(O R=1)$ refers to workers with low chemical exposure and negative hepatitis virus infection.

$\ddagger$ The referent group $(O R=1)$ refers to workers with low chemical exposure and positive hepatitis virus infection.

Hepatitis B and/or C infection have been associated with increased serum aminotransferase activities. ${ }^{11}$ Our results reveal similar findings, although the association between anti-HCV and ALT was not statistically significant. This is probably due to small numbers of workers with anti-HCV. Increased BMI is also associated with increased ALT and AST in our study (table 2). Increased BMI is a common aetiology of abnormal liver function tests for healthy workers. ${ }^{6712}$ Our results corroborate such an association. Alcohol consumption has been reported to be associated with abnormal liver function, ${ }^{11}$ but our results did not show this relation. This is most likely due to relative small amount of alcohol consumption by these workers. Lack of association between alcohol consumption with abnormal ALT and AST was also observed in several studies conducted in Taiwanese workers. ${ }^{10}{ }^{13}$ A study conducted in Italy showed that those who consumed $80 \mathrm{~g}$ each day had a greater risk of developing abnormal liver function among chronic symptomless HBV carriers. ${ }^{2}$ Positive synergism was also observed between HCV infection and alcohol consumption. ${ }^{3}$ Our study also revealed that HBV and HCV infection exacerbated the effect of alcohol on AST, although the effects of alcohol on ALT was less prominent. Again, this could result from the small amount of alcohol consumption in our study subjects. Additionally, AST increase is usually more prominent than ALT in alcoholic hepatitis. ${ }^{14} 15$ Thus, the relation between abnormal serum aminotransferases and non-occupational factors in our study is consistent with previous studies.

A recent study also showed that the relation between occupational dimethylformamide exposure and abnormal liver function was enhanced in those with HBV infection. ${ }^{13}$ Here, we showed that VCM and EDC together could also have a more than additive interaction with HBV and HCV infection. Further analysis in our study indicated that workers with HBeAg were more likely to have abnormal ALT and AST compared to those with HBsAg alone, when they were exposed to occupational chemicals. As both VCM and EDC were reported to be hazardous to the liver, detection
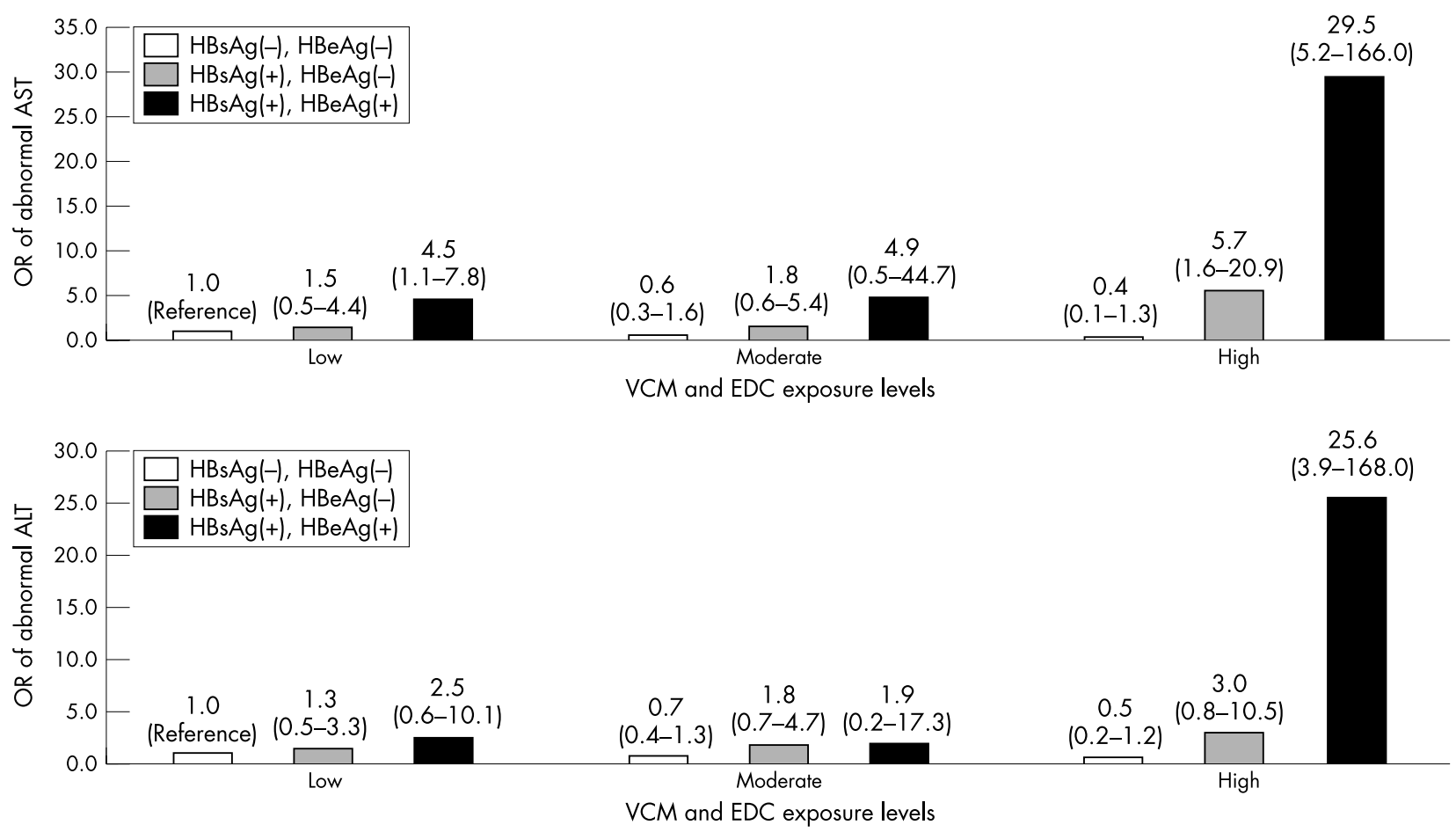

Figure 1 Odds ratio (OR) with 95\% confidence intervals (Cl) of abnormal AST (top) and ALT (bottom) among workers with different categories of exposure and hepatitis $\mathrm{B}$ infection; adjusted for age, drinking, anti-HCV, and BMI. The reference group $(O R=1.0)$ refers to workers with low chemical exposure and negative hepatitis $B$ infection. 
of such an effect is not surprising, and the mechanism should be clarified.

Previous human and animal studies indicated that glutathione (GSH) depletion can be caused by hepatitis virus infection. ${ }^{16-18}$ Glutathione S-transferases (GST) and glutathione play an important role in the metabolism of EDC and VCM, of which the electrophilic intermediate metabolites are conjugated with GSH to be detoxified..$^{19} 20$ Thus, GSH depletion caused by hepatitis virus infection may lead to an accumulation of active intermediate metabolites of EDC and VCM, then exacerbate EDC and VCM induced hepatotoxicity. Previous studies conducted with 1,1-dichloroethylene which showed correlations between hepatocellular damage and magnitudes of both covalent binding and GSH depletion also supported this proposed hypothesis. ${ }^{21}$ Our recent study also suggests that the GSTTl genotype may play an important role in liver aminotransferase abnormality caused by vinyl chloride. ${ }^{6}$ In patients with positive $\mathrm{HBeAg}$, there is more active HBV replication and inflammation, which can reduce the level of $\mathrm{GSH}^{22}$ Therefore, they are at a high risk of showing increases in AST and ALT, as shown in fig 1. We conclude that subjects with hepatitis B and/or C virus infections are more likely to be damaged by hepatotoxic agents; such a potential synergistic effect may be caused by GSH depletion after hepatitis virus infection.

Studies conducted in humans and rats also found that ethanol significantly decreased glutathione concentrations, ${ }^{23-25}$ of which the synergistic hepatotoxic effect between alcohol and hepatitis virus infection could also have resulted from potential overloading of the oxidative damage through the generation of reactive intermediates and decreased radical scavenging.

We are concerned that VCM or EDC workers with HBV and/or HCV infections may not be well protected under current occupational standards. We suggest that workers with HBeAg should not be exposed to hepatotoxins in their work. We also advise workers with anti-HCV and abnormal serum aminotransferases, not to be exposed to hepatotoxins. Serum aminotransferases in workers with positive HBsAg but negative HBeAg, need to be closely monitored if exposed to higher levels of VCM or EDC. Furthermore, a more stringent occupational standard is needed to protect workers exposed to hepatotoxins in countries where hepatitis $B$ and $C$ virus infection is prevalent.

\section{ACKNOWLEDGEMENTS}

We thank Mr Yen-Cheng Chen and Dr Chung-Li Du for technical assistance. We also thank Professor Robert Chen for editorial assistance. This study was supported by grants NSC 90-2320-B002-125 from the National Science Council, Taiwan.

\section{Authors' affiliations}

H-I Hsieh, Department of Family Medicine, Cathay General Hospital, Taipei, Taiwan

J-D Wang, P-C Chen, T-J Cheng, Institute of Occupational Medicine and Industrial Hygiene, College of Public Health, National Taiwan University, Taipei, Taiwan

\section{REFERENCES}

1 Chen JD, Wang JD, Tsai SY, et al. Effects of occupational and nonoccupational factors on liver function tests in workers exposed to solvent mixtures. Arch Environ Health 1997;52:270-4.

2 Villa E, Rubbiani L, Barchi T, et al. Susceptibility of chronic symptomless $\mathrm{HBsAg}$ carriers to ethanol-induced hepatic damage. Lancet 1982;2:1243-4.

3 Nevins CL, Malaty $\mathrm{H}$, Velez ME, et al. Interaction of alcohol and hepatitis $C$ virus infection on severity of liver disease. Dig Dis Sci 1999:44: 1236-42.

4 Chen DS. Natural history of chronic hepatitis B virus infection: new light on old story. J Gastroenterol Hepatol 1993;8:470-5.

5 Huang KY, Lin SR. Nationwide vaccination: a success story in Taiwan. Vaccine 2000;18:S35-8.

6 Huang CY, Huang KL, Cheng TJ, et al. The GSTT1 and CYP2E1 genotypes are possible factors causing vinyl chloride induced abnormal liver function. Arch Toxicol 1997;71:482-8.

7 Cheng TJ, Huang ML, You NC, et al. Abnormal liver function in workers exposed to low levels of ethylene dichloride and vinyl chloride monomer. J Occup Environ Med 1999;41:1128-33.

8 Chu CM, Sheen IS, Lin SM, et al. Sex difference in chronic hepatitis B virus infection: studies of serum $\mathrm{HBeAg}$ and alanine aminotransferase levels in 10,431 asymptomatic Chinese HBsAg carriers. Clin Infect Dis 1993;16:709-13.

9 Cheng TJ, Chou PY, Huang ML, et al. Increased lymphocyte sister chromatid exchange frequency in workers with exposure to low level of ethylene dichloride. Mutat Res 2000:470:109-14.

10 Du CL, Kuo ML, Chang HL, et al. Changes in lymphocyte single strand breakage and liver function of workers exposed to vinyl chloride monomer. Toxicol Lett 1995;77:379-85.

11 Pratt DS, Kaplan MM. Evaluation of abnormal liver-enzyme results in asymptomatic patients. N Engl J Med 2000;342:1266-71.

12 Salvaggio A, Periti M, Miano L, et al. Body mass index and liver enzyme activity in serum. Clin Chem 1991;37:720-3.

13 Luo JC, Kuo HW, Cheng TJ, et al. Abnormal liver function associated with occupational exposure to dimethylformamide and hepatitis B virus. J Occup Environ Med 2001;43:474-82.

14 Clermont RJ, Chalmers TC. The transaminase tests in liver disease. Medicine 1967;46:197-207.

15 Sorbi D, Boynton J, Lindor KD. The ratio of aspartate aminotransferase to alanine aminotransferase: potential value in differentiating nonalcoholic steatohepatitis from alcoholic liver disease. Am J Gastroenterol steatohepatitis from

16 Swietek K, Juszczyk J. Reduced glutathione concentration in erythrocytes of patients with acute and chronic viral hepatitis. J Viral Hepat 1997:4:139-41.

17 Barbaro G, Di Lorenzo G, Soldini M, et al. Hepatic glutathione deficiency in chronic hepatitis $C$ : quantitative evaluation in patients who are HIV positive and HIV negative and correlations with plasmatic and lymphocytic concentrations and with the activity of the liver disease. Am J Gastroenterol 1996;91:2569-73.

18 De Flora S, Hietanen $\mathrm{E}$, Bartsch $\mathrm{H}$, et al. Enhanced metabolic activation of chemical hepatocarcinogens in woodchucks infected with hepatitis $B$ virus. Carcinogenesis 1989;10:1099-106.

19 ATSDR (Agency for Toxic Substances and Disease Registry). Toxicological profile for vinyl chloride-update. Atlanta, GA: ATSDR, 1997.

20 World Health Organisation. Environmental health criteria 176. 1,2Dichloroethane. 2nd ed Geneva: WHO, 1995.

21 Dowsley TF, Forkert PG, Benesch LA, et al. Reaction of glutathione with the electrophilic metabolites of 1,1-dichloroethylene. Chem Biol Interact 1995:95:227-44.

22 Kaneko S, Miller RH, Di Bisceglie AM, et al. Detection of hepatitis B virus DNA in serum by polymerase chain reaction. Application for clinical diagnosis. Gastroenterology 1990;99:799-804.

23 Michelet F, Gueguen R, Leroy $\mathrm{P}$, et al. Blood and plasma glutathione measured in healthy subjects by HPLC: relation to sex, aging, biological variables, and life habits. Clin Chem 1995;41:1509-17.

$24 \mathrm{Oh} \mathrm{SI}, \mathrm{Kim} \mathrm{Cl}, \mathrm{Chun} \mathrm{HJ}$, et al. Chronic ethanol consumption affects glutathione status in rat liver. I Nutr 1998;128:758-63.

25 Vendemiale G, Grattagliano I, Signorile A, et al. Ethanol-induced changes of intracellular thiol compartmentation and protein redox status in the rat liver: effect of tauroursodeoxycholate. J Hepatol 1998:28:46-53. 